\title{
LOG-CONCAVITY OF ASYMPTOTIC MULTIGRADED HILBERT SERIES
}

\author{
ADAM MCCABE AND GREGORY G. SMITH
}

(Communicated by Irena Peeva)

\begin{abstract}
We study the linear map sending the numerator of the rational function representing the Hilbert series of a module to that of its $r$-th Veronese submodule. We show that the asymptotic behaviour as $r$ tends to infinity depends on the multidegree of the module and the underlying positively multigraded polynomial ring. More importantly, we give a polyhedral description for the asymptotic polynomial and prove that the coefficients are log-concave.
\end{abstract}

\section{INTRODUCTION}

Although motivated by multigraded Hilbert series, our main result only involves linear operators on a multivariate power series. To be explicit, let $A:=\left[\begin{array}{lll}\boldsymbol{a}_{1} & \cdots & \boldsymbol{a}_{n}\end{array}\right]$ be an integer $(d \times n)$-matrix of rank $d$ such that the only nonnegative vector in the kernel is the zero vector (i.e. $\boldsymbol{a}_{1}, \ldots, \boldsymbol{a}_{n}$ is an acyclic vector configuration; see [Zie, $\S 6.2])$. Equivalently, the rational function $1 /\left(\prod_{1 \leqslant j \leqslant n}\left(1-\boldsymbol{t}^{\boldsymbol{a}_{j}}\right)\right)$ has a unique expansion as a multivariate formal power series; cf. Lemma 8.16 in [MS]. For each positive integer $r$, consider the linear operator $\Phi_{r}$ induced by sifting out all terms with the exponent vector divisible by $r$ in the power series expansion of a rational function. More precisely, $\Phi_{r}$ acts on $F(\boldsymbol{t}) \in \mathbb{Z}\left[\boldsymbol{t}^{ \pm 1}\right]:=\mathbb{Z}\left[t_{1}, t_{1}^{-1}, \ldots, t_{d}, t_{d}^{-1}\right]$ as follows:

$$
\text { if } \frac{F(\boldsymbol{t})}{\prod_{1 \leqslant j \leqslant n}\left(1-\boldsymbol{t}^{\boldsymbol{a}_{j}}\right)}=\sum_{\boldsymbol{w}} c_{\boldsymbol{w}} \boldsymbol{t}^{\boldsymbol{w}}, \text { then } \sum_{\boldsymbol{w}} c_{r \boldsymbol{w}} \boldsymbol{t}^{\boldsymbol{w}}=\frac{\Phi_{r}[F(\boldsymbol{t})]}{\prod_{1 \leqslant j \leqslant n}\left(1-\boldsymbol{t}^{\boldsymbol{a}_{j}}\right)} .
$$

The goal of this article is to understand $\Phi_{r}[F(\boldsymbol{t})]$ for $r \gg 0$.

To state our result, $\alpha: \mathbb{R}^{n} \rightarrow \mathbb{R}^{d}$ denotes the linear map determined by $A$. Let $Z$ be the associated zonotope; it is the image under $\alpha$ of the unit hypercube $[0,1]^{n} \subset \mathbb{R}^{n}$. For each $\boldsymbol{u} \in \mathbb{Z}^{d}$, set $P(\boldsymbol{u}):=\alpha^{-1}(\boldsymbol{u}) \cap[0,1]^{n}$. We say that the map $\alpha$ is degenerate if there exists $\boldsymbol{u}$ in the boundary of $Z$ such that $\operatorname{dim} P(\boldsymbol{u})=$ $n-d$. By identifying the rational polytope $P(\boldsymbol{u})$ with a translate $P(\boldsymbol{u})+\boldsymbol{x}$ lying in $\operatorname{ker}(A)=\alpha^{-1}(\mathbf{0})$, the normalized volume $\operatorname{vol}_{n-d}(P(\boldsymbol{u}))$ equals $(n-d)$ ! times the Euclidean volume of $P(\boldsymbol{u})+\boldsymbol{x}$ with respect to the lattice $\alpha^{-1}(\mathbf{0}) \cap \mathbb{Z}^{n}$. Let $m$ be the greatest common divisor of the maximal minors of $A$; in other words, the sublattice $\mathbb{Z} A \subseteq \mathbb{Z}^{d}$ generated by the columns of $A$ has index $m$.

Received by the editors September 20, 2011.

2010 Mathematics Subject Classification. Primary 05E40, 13D40, 52B20.

(C) 2012 American Mathematical Society Reverts to public domain 28 years from publication 
Theorem 1.1. If $\alpha$ is nondegenerate and $F(\boldsymbol{t})=\sum_{\boldsymbol{v} \in \mathbb{Z} A} f_{\boldsymbol{v}} \boldsymbol{t}^{\boldsymbol{v}} \in \mathbb{Z}\left[\boldsymbol{t}^{ \pm 1}\right]$, then we have

$\limsup _{r \rightarrow \infty} \frac{\Phi_{r}[F(\boldsymbol{t})]}{r^{n-d}}=\left(\frac{F(\mathbf{1})}{(n-d) !}\right) K_{A}(\boldsymbol{t})$, where $K_{A}(\boldsymbol{t}):=\sum_{\boldsymbol{u} \in \operatorname{int}(Z) \cap \mathbb{Z}^{d}} \operatorname{vol}_{n-d}(P(\boldsymbol{u})) \boldsymbol{t}^{\boldsymbol{u}}$.

Moreover, the coefficients of $K_{A}(\boldsymbol{t})$ are log-concave, quasi-concave, and sum to $m^{n-d}(n-d) !$.

When $A=[1 \cdots 1]$, Theorem 1.1 specializes to Theorem 5.1 in DF2]. In this case, $K_{A}(\boldsymbol{t})$ is the Eulerian polynomial $\sum_{i \geqslant 0}\left\langle\begin{array}{c}n-1 \\ i\end{array}\right\rangle t^{i+1}$, where $\left\langle\begin{array}{c}n-1 \\ i\end{array}\right\rangle$ counts the permutations of $\{1, \ldots, n-1\}$ with exactly $i$ ascents. If $A$ is a totally unimodular matrix (i.e. each subdeterminant of $A$ is \pm 1 or 0 ), then we have $K_{A}(\boldsymbol{t}) \in \mathbb{Z}\left[\boldsymbol{t}^{ \pm 1}\right]$; see Remark 2.2. Thus, for any appropriate matrix $A$, one might regard $K_{A}(\boldsymbol{t})$ as a generalization of the Eulerian polynomial. However, $K_{A}(\boldsymbol{t})$ is not obviously related to the multivariate Eulerian polynomials in [BHVW] $\S 4.3$ ] or the mixed Eulerian numbers in [Pos, §16]. Nevertheless, the proof of Theorem 1.1 (see Step 1 in \$2) implies that, for any $F(\boldsymbol{t})=\sum_{\boldsymbol{v} \in \operatorname{int}(m Z)} f_{\boldsymbol{v}} \boldsymbol{t}^{\boldsymbol{v}} \in \mathbb{Z}\left[\boldsymbol{t}^{ \pm 1}\right]$ satisfying $F(\mathbf{1})>0$, there exists $r_{0} \in \mathbb{N}$ such that, for all $r \geqslant r_{0}, \Phi_{m r}[F(\boldsymbol{t})]$ has nonnegative coefficients that are both log-concave and quasi-concave. Since quasi-concavity is the multivariate version of unimodality (see Step 2 in 92 ), Theorem 1.1 generalizes those parts of Theorem 1.4 in BW] and Theorem 1.2 in [BS that do not make explicit reference to the roots of $F(\boldsymbol{t})$. It is an open problem to effectively bound $r_{0}$.

Despite sharing most properties of the Eulerian polynomial, the Laurent polynomial $K_{A}(\boldsymbol{t})$ need not satisfy the multivariate analogues of real-zero univariate polynomials. For example, if

$$
A=\left[\begin{array}{rrrrr}
1 & -1 & 1 & 0 & 0 \\
0 & 1 & -1 & 1 & 0 \\
0 & 0 & 1 & -1 & 1
\end{array}\right]
$$

then we have $K_{A}\left(t_{1}, t_{2}, t_{3}\right)=t_{1} t_{3}+t_{2}$, which does not have the half-plane property; see Theorem 3.2 in Bra. Notably, the polynomial $K_{A}\left(t_{1}, t_{2}, t_{3}\right)=t_{1} t_{3}+t_{2}$ is neither real stable nor Hurwitz stable.

In contrast with $\mathrm{BW}$, $\mathrm{BS}$, or $\mathrm{DF} 2$, we provide a new proof that the Eulerian numbers $\left\langle\begin{array}{c}n \\ i\end{array}\right\rangle$ are log-concave and unimodal. We use polyhedral geometry and the Prékopa-Leindler inequality (a.k.a. the functional form of the Brunn-Minkowski inequality) to establish the log-concavity for the coefficients of $K_{A}(\boldsymbol{t})$; see Proposition 2.1. Since this method does not appear in the survey articles [Sta2, Bre, our approach to log-concavity appears to be novel within algebraic combinatorics.

Motivation. Our primary motivation for Theorem 1.1 comes from commutative algebra and algebraic geometry. The matrix $A$ defines a positive $\mathbb{Z}^{d}$-grading on the polynomial ring $S:=\mathbb{C}\left[x_{1}, \ldots, x_{n}\right]$ by setting $\operatorname{deg}\left(x_{j}\right):=\boldsymbol{a}_{j}$ for all $1 \leqslant j \leqslant n$; see Definition 8.7 in [MS]. For any finitely generated $\mathbb{Z}^{d}$-graded $S$-module $M=$ $\bigoplus_{\boldsymbol{w} \in \mathbb{Z}^{d}} M_{\boldsymbol{w}}$, the Hilbert series of $M$ can be expressed uniquely as a rational function of the form $F(\boldsymbol{t}) /\left(\prod_{1 \leqslant j \leqslant n}\left(1-\boldsymbol{t}^{\boldsymbol{a}_{j}}\right)\right)$; see Theorem 8.20 in [MS. The numerator $F(t)$ is the Poincaré polynomial or K-polynomial of $M$ - it records the alternating sum of the multigraded Betti numbers for $M$; see Definition 8.21 and Theorem 8.23 in [MS]. Applying the operator $\Phi_{r}$ to the numerator of Hilbert series for $M$ yields the numerator of Hilbert series for the $r$-th Veronese submodule $\bigoplus_{\boldsymbol{w} \in \mathbb{Z}^{d}} M_{r \boldsymbol{w}}$. Theorem 1.1 shows that there is a unique asymptotic $K$-polynomial 
for each $M$; see 93 for the important case in which $F(\mathbf{1})=0$. Therefore, Theorem 1.1 gives some information about the solution to Problem 5.3 in EL. The results in both [EL and this paper suggest that the asymptotic structure is surprisingly insensitive to the specific module.

Geometrically, the $\mathbb{Z}^{d}$-grading on $S$ corresponds to the action of the torus $\left(\mathbb{C}^{*}\right)^{d}$ on $\mathbb{A}^{n}$, and a multigraded $S$-module $M$ corresponds to a torus-equivariant sheaf on $\mathbb{A}^{n}$. In this setting, the numerator $F(\boldsymbol{t})$ is the class in the equivariant $K$-theory of $\mathbb{A}^{n}$ represented by the corresponding sheaf; see Theorem 8.34 in MS. Hence, Theorem 1.1 implies that the Veronese operator $\Phi_{r}$ distinguishes the class $K_{A}(\boldsymbol{t})$ within equivariant $K$-theory. By taking a GIT quotient (e.g [MS, §10.4]), we see that the $(n-d)$-dimensional toric variety $\mathbb{A}^{n} /\left(\mathbb{C}^{*}\right)^{d}$ is equipped with a distinguished $K$-theory class $K_{A}(\boldsymbol{t})$. A geometric explanation for this distinguished $K$-theory class would be interesting.

Our secondary motivation comes from the theory of lattice point enumeration. Given lattice polytopes $P_{1}, \ldots, P_{d}$ in $\mathbb{R}^{n}$ and nonnegative integers $w_{1}, \ldots, w_{d}$, the number of lattice points in the Minkowski sum of the $w_{i}$-dilates of the $P_{i}$ is denoted $\left|\left(w_{1} P_{1}+\cdots+w_{d} P_{d}\right) \cap \mathbb{Z}^{d}\right|$. Ehrhart theory implies that the generating series $\sum_{\left(w_{1}, \ldots, w_{d}\right)}\left|\left(w_{1} P_{1}+\cdots+w_{d} P_{d}\right) \cap \mathbb{Z}^{d}\right| t_{1}^{w_{1}} \cdots t_{d}^{w_{d}}$ can be expressed in the form $F(\boldsymbol{t}) /\left(\prod_{1 \leqslant j \leqslant n}\left(1-\boldsymbol{t}^{\boldsymbol{a}_{j}}\right)\right)$. The numerator $F(\boldsymbol{t})$ is a multivariate $h^{*}$-vector (a.k.a. Ehrhart $h$-vector or $\delta$-polynomial) for the collection $P_{1}, \ldots, P_{d}$. Thus, Theorem 1.1 also yields a multivariate analogue of Corollary 1.3 in [BS].

Multivariate formal power series of the form $F(\boldsymbol{t}) /\left(\prod_{1 \leqslant j \leqslant n}\left(1-\boldsymbol{t}^{\boldsymbol{a}_{j}}\right)\right)$ also arise naturally in many other areas of mathematics. Perhaps the most ubiquitous source is the vector partition function $\psi_{A}: \mathbb{N}^{d} \rightarrow \mathbb{N}$ associated to $A ; \psi_{A}(\boldsymbol{u})$ counts the number of nonnegative integer vectors $\boldsymbol{x} \in \mathbb{N}^{n}$ such that $A \boldsymbol{x}=\boldsymbol{u}$. The generating series $\sum_{\boldsymbol{u}} \psi_{A}(\boldsymbol{u}) \boldsymbol{t}^{\boldsymbol{u}}$ equals $1 /\left(\prod_{1 \leqslant j \leqslant n}\left(1-\boldsymbol{t}^{\boldsymbol{a}_{j}}\right)\right)$. As $[\mathrm{Stu}$ indicates, vector partition functions appear in representation theory, approximation theory, and statistics. Reinterpreting $\Phi_{r}$ in each of these areas will yield new insights into $K_{A}(\boldsymbol{t})$.

Basic examples. We end this section with two examples illustrating Theorem 1.1

Example 1.2. Let $A=\left[\begin{array}{lll}2 & 1 & 0 \\ 0 & 1 & 2\end{array}\right]$, so that $d=2, n=3$, and $m=\operatorname{gcd}(2,4,2)=2$. For all $r>2$, we have $\Phi_{2 r}[1]=(r-1) t_{1}^{2} t_{2}^{2}+(r-1) t_{1}^{2} t_{2}+(r-1) t_{1} t_{2}^{2}+r t_{1} t_{2}+t_{1}+t_{2}+1$, which implies that $\lim _{r \rightarrow \infty} \frac{\Phi_{2 r}[1]}{2 r}=\frac{1}{2} t_{1}^{2} t_{2}^{2}+\frac{1}{2} t_{1}^{2} t_{2}+\frac{1}{2} t_{1} t_{2}^{2}+\frac{1}{2} t_{1} t_{2}$. However, we also have $\Phi_{2 r+1}[1]=r t_{1}^{2} t_{2}^{2}+r t_{1} t_{2}+1$, which shows that $\lim _{r \rightarrow \infty} \frac{\Phi_{r}[1]}{r}$ does not exist. Nevertheless, we obtain $\limsup _{r \rightarrow \infty} \frac{\Phi_{r}[1]}{r}=\frac{1}{2} t_{1}^{2} t_{2}^{2}+\frac{1}{2} t_{1}^{2} t_{2}+\frac{1}{2} t_{1} t_{2}^{2}+\frac{1}{2} t_{1} t_{2}$.

The zonotope $Z$ associated to $A$ is $\operatorname{conv}\{(0,0),(2,0),(0,2),(3,1),(1,3),(3,3)\}$. Its interior lattice points are $(1,1),(2,1),(1,2),(2,2)$ and the associated polytopes are the line segments:

$$
\begin{array}{ll}
P(1,1)=\operatorname{conv}\left\{(0,1,0),\left(\frac{1}{2}, 0, \frac{1}{2}\right)\right\}, & P(2,1)=\operatorname{conv}\left\{\left(\frac{1}{2}, 1,0\right),\left(1,0, \frac{1}{2}\right)\right\}, \\
P(1,2)=\operatorname{conv}\left\{\left(0,1, \frac{1}{2}\right),\left(\frac{1}{2}, 0,1\right)\right\}, & P(2,2)=\operatorname{conv}\left\{(1,0,1),\left(\frac{1}{2}, 0,1\right)\right\} .
\end{array}
$$

Since $\alpha^{-1}(\mathbf{0}) \cap \mathbb{Z}^{3}=\{(i,-2 i, i): i \in \mathbb{Z}\}$ and the coefficient of $\boldsymbol{t}^{\boldsymbol{u}}$ in $K_{A}(\boldsymbol{t})$ is the normalized volume of $P(\boldsymbol{u})$, we have $K_{A}\left(t_{1}, t_{2}\right)=\frac{1}{2} t_{1}^{2} t_{2}^{2}+\frac{1}{2} t_{1}^{2} t_{2}+\frac{1}{2} t_{1} t_{2}^{2}+\frac{1}{2} t_{1} t_{2}$. The coefficients sum to 2 . 
Example 1.3. Let $A=\left[\begin{array}{lll}1 & 1 & 0 \\ 0 & 0 & 1\end{array}\right]$, so that $d=2, n=3$, and $m=1$. It follows that

$$
\begin{array}{cc}
\limsup _{r \rightarrow \infty} \frac{\Phi_{r}[1]}{r}=\lim _{r \rightarrow \infty} \frac{(r-1) t_{1}+1}{r}=t_{1}, & \limsup _{r \rightarrow \infty} \frac{\Phi_{r}\left[t_{1}\right]}{r}=\lim _{r \rightarrow \infty} \frac{(r-1) t_{1}}{r}=t_{1}, \\
\limsup _{r \rightarrow \infty} \frac{\Phi_{r}\left[t_{2}\right]}{r}=\lim _{r \rightarrow \infty} \frac{(r-1) t_{1} t_{2}+t_{2}}{r}=t_{1} t_{2}, & \limsup _{r \rightarrow \infty} \frac{\Phi_{r}\left[t_{1} t_{2}\right]}{r}=\lim _{r \rightarrow \infty} \frac{r t_{1} t_{2}}{r}=t_{1} t_{2} .
\end{array}
$$

Although each of the limits exists, they do not simply depend up to a scalar on the matrix $A$. The zonotope $Z=\operatorname{conv}\{(0,0),(2,0),(0,1),(2,1)\}$ has no interior lattice points, but both of the polytopes $P(1,0)=\operatorname{conv}\{(1,0,0),(0,1,0)\}$ and $P(1,1)=\operatorname{conv}\{(1,0,1),(0,1,1)\}$ have dimension $n-d=1$. In particular, the map $\alpha$ is degenerate.

Further examples, open problems, and other connections are discussed in 93 . The proof of Theorem 1.1 is given in $乌 2$.

\section{THE PROOF OF THE MAIN THEOREM}

We divide the proof of Theorem 1.1 into three steps: Step 1 establishes that the limit exists and provides a polyhedral interpretation for $K_{A}(\boldsymbol{t})$, Step 2 uses the polyhedral interpretation to prove the log-concavity of the coefficients, and Step 3 gives a geometric explanation for the sum of the coefficients. For brevity, we write $\prod_{j}:=\prod_{1 \leqslant j \leqslant n}$ and $\sum_{j}:=\sum_{1 \leqslant j \leqslant n}$.

Step 1. To begin, we describe the matrix associated to the linear operator $\Phi_{r}$. Despite being defined via multivariate formal power series, $\Phi_{r}$ may be understood in terms of a linear operator on Laurent polynomials. Specifically, let $\Psi_{r} \in$ $\operatorname{End}\left(\mathbb{Z}\left[\boldsymbol{t}^{ \pm 1}\right]\right)$ be the linear operator that discards the terms with exponent vectors that are not componentwise divisible by $r$ and divides each of the remaining exponent vectors by $r$. Consider a Laurent polynomial $F(\boldsymbol{t}) \in \mathbb{Z}\left[\boldsymbol{t}^{ \pm 1}\right]$ such that $F(\boldsymbol{t}) /\left(\prod_{j}\left(1-\boldsymbol{t}^{\boldsymbol{a}_{j}}\right)\right)=\sum_{\boldsymbol{w}} c_{\boldsymbol{w}} \boldsymbol{t}^{\boldsymbol{w}}$. The linear operator $\Psi_{r}$ lifts to an endomorphism on multivariate formal power series. Applying $\Psi_{r}$ to this rational function yields

$$
\begin{aligned}
\frac{\Phi_{r}[F(\boldsymbol{t})]}{\prod_{j}\left(1-\boldsymbol{t}^{\boldsymbol{a}_{j}}\right)}=\sum_{\boldsymbol{w}} c_{r \boldsymbol{w}} \boldsymbol{t}^{\boldsymbol{w}} & =\Psi_{r}\left[\frac{F(\boldsymbol{t})}{\prod_{j}\left(1-\boldsymbol{t}^{\boldsymbol{a}_{j}}\right)}\right] \\
& =\Psi_{r}\left(\frac{F(\boldsymbol{t}) \prod_{j}\left(1+\boldsymbol{t}^{\boldsymbol{a}_{j}}+\boldsymbol{t}^{2 \boldsymbol{a}_{j}}+\cdots+\boldsymbol{t}^{(r-1) \boldsymbol{a}_{j}}\right)}{\prod_{j}\left(1-\boldsymbol{t}^{r \boldsymbol{a}_{j}}\right)}\right) \\
& =\frac{\Psi_{r}\left[F(\boldsymbol{t}) \prod_{j}\left(1+\boldsymbol{t}^{\boldsymbol{a}_{j}}+\boldsymbol{t}^{2 \boldsymbol{a}_{j}}+\cdots+\boldsymbol{t}^{(r-1) \boldsymbol{a}_{j}}\right)\right]}{\prod_{j}\left(1-\boldsymbol{t}^{\boldsymbol{a}_{j}}\right)} .
\end{aligned}
$$

Hence, we have $\Phi_{r}[F(\boldsymbol{t})]=\Psi_{r}\left[F(\boldsymbol{t}) \prod_{j}\left(1+\boldsymbol{t}^{\boldsymbol{a}_{j}}+\boldsymbol{t}^{2 \boldsymbol{a}_{j}}+\cdots+\boldsymbol{t}^{(r-1) \boldsymbol{a}_{j}}\right)\right]$; this is a multivariate version of Lemma 3.2 in [BS]. To express $\Phi_{r}$ as a matrix with respect to the monomial basis, set

$$
C_{r}(\boldsymbol{u}, \boldsymbol{v}):=\left|\left\{\boldsymbol{x}=\left(x_{1}, \ldots, x_{n}\right) \in \mathbb{Z}^{n} \cap[0, r-1]^{n}: \sum_{j} x_{j} \boldsymbol{a}_{j}=r \boldsymbol{u}-\boldsymbol{v}\right\}\right| .
$$

Since $F(\boldsymbol{t}):=\sum_{\boldsymbol{v} \in \mathbb{Z} A} f_{\boldsymbol{v}} \boldsymbol{t}^{\boldsymbol{v}}$, we have $\Phi_{r}[F(\boldsymbol{t})]=\sum_{\boldsymbol{u}}\left(\sum_{\boldsymbol{v} \in \mathbb{Z} A} C_{r}(\boldsymbol{u}, \boldsymbol{v}) f_{\boldsymbol{v}}\right) \boldsymbol{t}^{\boldsymbol{u}}$. As $v \in \mathbb{Z} A$, we also have $C_{r}(\boldsymbol{u}, \boldsymbol{v})=0$ for all $\boldsymbol{u} \notin \mathbb{Z} A$. However, the sublattice $\mathbb{Z} A \subseteq \mathbb{Z}^{d}$ has index $m$, so $m \boldsymbol{u} \in \mathbb{Z} A$. Hence, it is enough to consider

$$
\Phi_{m r}[F(\boldsymbol{t})]=\sum_{\boldsymbol{u}}\left(\sum_{\boldsymbol{v} \in \mathbb{Z} A} C_{m r}(\boldsymbol{u}, \boldsymbol{v}) f_{\boldsymbol{v}}\right) \boldsymbol{t}^{\boldsymbol{u}} .
$$


We next relate the integer coefficients $C_{m r}(\boldsymbol{u}, \boldsymbol{v})$ to the normalized volume of the rational polytope $P(\boldsymbol{u}):=\left\{\boldsymbol{x} \in[0,1]^{n}: \sum_{j} x_{j} \boldsymbol{a}_{j}=\boldsymbol{u}\right\}=\alpha^{-1}(\boldsymbol{u}) \cap[0,1]^{n} \subseteq \mathbb{R}^{n}$. For any $\boldsymbol{u} \in \mathbb{Z}^{d}$ and any $\boldsymbol{v} \in \mathbb{Z} A$, there exists $\boldsymbol{y}$ and $\boldsymbol{z} \in \mathbb{Z}^{n}$ such that $m \boldsymbol{u}=\alpha(\boldsymbol{y})$ and $\boldsymbol{v}=\alpha(\boldsymbol{z})$. It follows that

$$
C_{m r}(\boldsymbol{u}, \boldsymbol{v})=\left|\left((m r-1) P(\boldsymbol{u})+\frac{1}{m} \boldsymbol{y}-\boldsymbol{z}\right) \cap \mathbb{Z}^{n}\right|=\left|\left((m r-1) P(\boldsymbol{u})+\frac{1}{m} \boldsymbol{y}\right) \cap \mathbb{Z}^{n}\right| .
$$

The enumerative theory for systems of linear diophantine equations establishes that the function

$r \mapsto\left|\left((m r-1) P(\boldsymbol{u})+\frac{1}{m} \boldsymbol{y}\right) \cap \mathbb{Z}^{n}\right|=\left|\left((r-1)(m P(\boldsymbol{u}))+(m-1) P(\boldsymbol{u})+\frac{1}{m} \boldsymbol{y}\right) \cap \mathbb{Z}^{n}\right|$

is a quasi-polynomial; see Theorem 6.50 in $\mathrm{BG}$ ] or Theorem 4.6.11 in Sta3. Moreover, the leading coefficient of this quasi-polynomial is the relative volume of $m P(\boldsymbol{u})$ with respect to the lattice $\alpha^{-1}(r m u) \cap \mathbb{Z}^{n}$; see Theorem 6.55 in $[\mathrm{BG}$. Since $m \boldsymbol{u} \in \mathbb{Z} A$ and $(m r-1) P(\boldsymbol{u})+\frac{1}{m} \boldsymbol{y} \subset \alpha^{-1}(r m u)$, the normalized volume $\operatorname{vol}_{n-d}(P(\boldsymbol{u}))$ equals $(n-d) ! / m^{n-d}$ times the coefficient of the degree $n-d$ term in this quasi-polynomial, so

$$
\lim _{r \rightarrow \infty} \frac{C_{m r}(\boldsymbol{u}, \boldsymbol{v})}{(m r)^{n-d}}=\lim _{r \rightarrow \infty} \frac{\left|\left((m r-1) P(\boldsymbol{u})+\frac{1}{m} \boldsymbol{y}\right) \cap \mathbb{Z}^{n}\right|}{(m r)^{n-d}}=\frac{\operatorname{vol}_{n-d}(P(\boldsymbol{u}))}{(n-d) !} .
$$

By hypothesis, $\alpha$ is nondegenerate, so $P(\boldsymbol{u})$ has dimension $n-d$ if and only if $\boldsymbol{u}$ lies in the interior of $Z$. Therefore, we have

$$
\begin{aligned}
\lim _{r \rightarrow \infty} \frac{\Phi_{m r}[F(\boldsymbol{t})]}{(m r)^{n-d}} & =\sum_{\boldsymbol{u}}\left(\sum_{\boldsymbol{v} \in \mathbb{Z} A}\left(\lim _{r \rightarrow \infty} \frac{C_{m r}(\boldsymbol{u}, \boldsymbol{v})}{(m r)^{n-d}}\right) f_{\boldsymbol{v}}\right) \boldsymbol{t}^{\boldsymbol{u}} \\
& =\frac{F(\mathbf{1})}{(n-d) !}\left(\sum_{\boldsymbol{u} \in \operatorname{int}(Z) \cap \mathbb{Z}^{d}} \operatorname{vol}_{n-d}(P(\boldsymbol{u})) \boldsymbol{t}^{\boldsymbol{u}}\right) .
\end{aligned}
$$

It follows immediately from this polyhedral description that $K_{A}(\boldsymbol{t})$ inherits the symmetries of the zonotope $Z$.

Step 2. The second step takes advantage of a well-known theorem from convex geometric analysis. To isolate the applicable result, recall that $g: \mathbb{R}^{d} \rightarrow \mathbb{R}$ is logconcave if $g(\boldsymbol{u})>0$ and $\log (g)$ is concave. Equivalently, for $s \in[0,1]$ and $\boldsymbol{u}, \boldsymbol{v} \in \mathbb{Z}^{d}$, we have the inequality $g(s \boldsymbol{u}+(1-s) \boldsymbol{v}) \geqslant g(\boldsymbol{u})^{s} g(\boldsymbol{v})^{1-s}$.

Proposition 2.1. Let $X \subset \mathbb{R}^{n}$ be a closed convex set and let $\pi: \mathbb{R}^{n} \rightarrow \mathbb{R}^{d}$ be a surjective linear map. Consider the function $g: \mathbb{R}^{d} \rightarrow \mathbb{R}$ that assigns to $\boldsymbol{u} \in \mathbb{R}^{d}$ the volume of the fibre $\pi^{-1}(\boldsymbol{u}) \cap X$. It follows that $g$ is log-concave.

Proof. Choosing a basis for $\operatorname{ker}(\pi)$ defines an injective linear map $\varphi: \mathbb{R}^{n-d} \rightarrow \mathbb{R}^{n}$ such that $\pi \circ \varphi$ is the zero map. We may also choose an injective linear map $\eta: \mathbb{R}^{d} \rightarrow \mathbb{R}^{n}$ such that $\pi \circ \eta$ is the identity map, because $\pi$ is surjective. For any $\boldsymbol{u} \in \mathbb{R}^{d}$, we set $X(\boldsymbol{u}):=\left\{\boldsymbol{p} \in \mathbb{R}^{n-d}: \varphi(\boldsymbol{p})+\eta(\boldsymbol{u}) \in X\right\} \cong \pi^{-1}(\boldsymbol{u}) \cap X$, so that $g(\boldsymbol{u})=\operatorname{vol}(X(\boldsymbol{u}))$. Since $X$ is convex, we have

$$
s X(\boldsymbol{u})+(1-s) X(\boldsymbol{v}) \subseteq X(s \boldsymbol{u}+(1-s) \boldsymbol{v})
$$

for all $s \in[0,1]$ and $\boldsymbol{u}, \boldsymbol{v} \in \mathbb{R}^{d}$. If $1_{Y}: \mathbb{R}^{n-d} \rightarrow \mathbb{R}$ denotes the indicator function for $Y \subseteq \mathbb{R}^{n-d}$, i.e. $1_{Y}(\boldsymbol{y})=\left\{\begin{array}{ll}1 & \text { if } \boldsymbol{y} \in Y \\ 0 & \text { if } \boldsymbol{y} \notin Y\end{array}\right.$, then we obtain

$$
1_{X(s \boldsymbol{u}+(1-s) \boldsymbol{v})}(s \boldsymbol{p}+(1-s) \boldsymbol{q}) \geqslant\left(1_{X(\boldsymbol{u})}(\boldsymbol{p})\right)^{s}\left(1_{X(\boldsymbol{v})}(\boldsymbol{q})\right)^{1-s}
$$


for all $\boldsymbol{p}, \boldsymbol{q} \in \mathbb{R}^{n-d}$. There exists a positive $k \in \mathbb{R}$ such that

$$
g(\boldsymbol{u})=k \int_{\mathbb{R}^{n-d}} 1_{X(\boldsymbol{u})}(\boldsymbol{p}) d \boldsymbol{p}
$$

so the Prékopa-Leindler inequality (Theorem 7.1 in [Gar] or Theorem 6.4 in [Vil]) yields

$$
\begin{aligned}
g(s \boldsymbol{u}+(1-s) \boldsymbol{v}) & =k \int_{\mathbb{R}^{n-d}} 1_{X(s \boldsymbol{u}+(1-s) \boldsymbol{v})}(\boldsymbol{p}) d \boldsymbol{p} \\
& \geqslant k\left(\int_{\mathbb{R}^{n-d}} 1_{X(\boldsymbol{u})}(\boldsymbol{p}) d \boldsymbol{p}\right)^{s}\left(\int_{\mathbb{R}^{n-d}} 1_{X(\boldsymbol{v})}(\boldsymbol{p}) d \boldsymbol{p}\right)^{1-s} \\
& =(g(\boldsymbol{u}))^{s}(g(\boldsymbol{v}))^{1-s}
\end{aligned}
$$

To establish that the coefficient function of $K_{A}(\boldsymbol{t})$ is log-concave, we simply apply Proposition 2.1 when $X=[0,1]^{n}$ and $\pi=\alpha$, because Step 1 shows that the coefficients of $K_{A}(\boldsymbol{t})$ equal the normalized volume of the fibres of the map $\alpha:[0,1]^{n} \rightarrow Z$.

We finish this step by showing that the coefficient function of $K_{A}(\boldsymbol{t})$ is quasiconcave. A function $g: \mathbb{R}^{d} \rightarrow \mathbb{R}$ is quasi-concave if, for all $e \in \mathbb{R}$, its superlevel set $\left\{\boldsymbol{u} \in \mathbb{R}^{d}: g(\boldsymbol{u}) \geqslant e\right\}$ is convex. Equivalently, for $s \in[0,1]$ and $\boldsymbol{u}, \boldsymbol{v} \in \mathbb{R}^{d}$, we have $g(s \boldsymbol{u}+(1-s) \boldsymbol{v}) \geqslant \min (g(\boldsymbol{u}), g(\boldsymbol{v}))$. In particular, every positive log-concave function is quasi-concave.

Step 3. Lastly, we reinterpret the coefficients of $K_{A}(\boldsymbol{t})$ as the normalized volumes of certain regions in the hypercube $[0, m]^{n-d}$. This perspective is inspired by the well-known interpretation for the Eulerian numbers as the normalized volume of "slabs" in the hypercube; see [Sta1]. In fact, when $A=[1 \cdots 1]$, we recover this interpretation.

By reordering the columns and making a unimodular change of basis on $\mathbb{Z}^{d}$, it suffices to consider the case in which $A=\left[H \mid B^{\prime}\right]$, where $H$ is a $(d \times d)$-matrix in Hermite normal form, $\operatorname{det}(H)=m$, and $B^{\prime}=\left[\boldsymbol{b}_{1} \cdots \boldsymbol{b}_{d}\right]^{\mathrm{t}}$ is a $(d \times(n-d))$-matrix; see [Sch, §4.1]. Let $B$ be the integer block $(n \times d)$-matrix $\left[\begin{array}{c}m H^{-1} B^{\prime} \\ -m I_{n-d}\end{array}\right]$, so that $A B=0$. If $m=1$, then $H=I_{d}$ and $B$ is the Gale dual of $A$; see [Zie, $\S 6.4$ ]. Thus, we may assume that $A$ has this block structure.

For $\boldsymbol{u} \in \mathbb{Z}^{d}$, consider regions

$$
R(\boldsymbol{u}):=\left\{\boldsymbol{p} \in[0, m]^{n-d}: m\left(u_{i}-1\right) \leqslant \boldsymbol{b}_{i} \cdot \boldsymbol{p} \leqslant m u_{i} \text { for all } 1 \leqslant i \leqslant d\right\} \subset \mathbb{R}^{n-d} .
$$

By definition, each region $R(\boldsymbol{u})$ is a rational polytope and, combined together, these regions partition the hypercube $[0, m]^{n-d}$. Hence, the union $\bigcup_{\boldsymbol{u}} R(\boldsymbol{u})$ has normalized volume equal to $m^{n-d}(n-d)$ !.

To complete the proof, it is enough to show that the coefficients of $K_{A}(\boldsymbol{t})$ also correspond to the normalized volumes of the regions $R(\boldsymbol{u})$. If $J$ is the integer $(n \times d)$-matrix $\left[\begin{array}{c}m H^{-1} \\ 0\end{array}\right]$, then we obtain $A J=m I_{d}$. Moreover, the inequalities $m\left(u_{i}-1\right) \leqslant \boldsymbol{b}_{i} \cdot \boldsymbol{p} \leqslant m u_{i}$ hold if and only if the inequalities $1 \geqslant u_{i}-\frac{1}{m} \boldsymbol{b}_{i} \cdot \boldsymbol{p} \geqslant 0$ hold. It follows that the affine map $\boldsymbol{p} \mapsto \frac{1}{m}(J \boldsymbol{u}-B \boldsymbol{p})$ sends the region $R(\boldsymbol{u})$ into the rational polytope $P(\boldsymbol{u})=\left\{\boldsymbol{x} \in[0,1]^{n}: \sum_{j} x_{j} \boldsymbol{a}_{j}=\boldsymbol{u}\right\}=\alpha^{-1}(\boldsymbol{u}) \cap[0,1]^{n}$. The inverse map is associated to the $((n-d) \times n)$-matrix $L:=\left[0 \mid I_{n-d}\right]$ which satisfies $L J=0$ and $L B=-m I_{n-d}$. Since Step 1 establishes that the coefficients 
of $K_{A}(\boldsymbol{t})$ equal the normalized volumes of the polytopes $P(\boldsymbol{u})$, we conclude that $K_{A}(\mathbf{1})=m^{n-d}(n-d)$ !, as required.

Remark 2.2. Suppose that $A$ is a totally unimodular matrix which implies that $m=1$. If $\boldsymbol{u} \in \mathbb{Z}^{d}$, then $P(\boldsymbol{u})$ is a lattice polytope (see Theorem 19.1 in [Sch]) and $C_{r}(\boldsymbol{u}, \boldsymbol{v})=\left|((r-1) P(\boldsymbol{u})) \cap \mathbb{Z}^{n}\right|$. Thus, the normalized volume $\operatorname{vol}_{n-d}(P(\boldsymbol{u}))$ is a nonnegative integer, which implies that $K_{A}(\mathbf{1})=(n-d)$ ! and $K_{A}(\boldsymbol{t}) \in \mathbb{Z}\left[\boldsymbol{t}^{ \pm 1}\right]$. Finding a combinatorial description for these coefficients remains an open problem. One tantalizing possibility is that the coefficients naturally enumerate some partition of the symmetric group on $n-d$ letters.

\section{OTHER CONNECTIONS}

This section uses two additional examples to highlight some potential applications for Theorem 1.1. To help orient future research, we also state a few open problems.

Asymptotic equalities. When $F(\mathbf{1}) \neq 0$, Theorem 1.1 shows that some of the coefficients of $\Phi_{r}[F(\boldsymbol{t})]$, regarded as functions of $r$, are asymptotically equal to an explicit multiple of $r^{n-d}$. In many situations, Theorem 1.1 yields an asymptotic equality even though $F(\mathbf{1})=0$. Roughly speaking, the Taylor expansion of $F(\boldsymbol{t})$ about $\boldsymbol{t}=\mathbf{1}$ allows one to cancel factors from the denominator and, thereby, apply Theorem 1.1 to a submatrix of $A$. We demonstrate this approach in the following prototypical example.

Example 3.1. Let $S=\mathbb{C}\left[x_{1}, \ldots, x_{6}\right]$ be the polynomial ring with the positive $\mathbb{Z}^{2}$ grading induced by $A=\left[\begin{array}{llllll}2 & 1 & 2 & 0 & 1 & 2 \\ 0 & 1 & 1 & 2 & 2 & 2\end{array}\right]$. Observe that $m=1$. Fix the finitely generated $\mathbb{Z}^{2}$-graded $S$-module $M=S / I$ where $I=\left\langle x_{5}^{2}-x_{4} x_{6}, x_{3} x_{5}-x_{2} x_{6}, x_{3} x_{4}-x_{2} x_{5}, x_{3}^{2}-\right.$ $\left.x_{1} x_{6}, x_{2} x_{3}-x_{1} x_{5}, x_{2}^{2}-x_{1} x_{6}\right\rangle$. Under the standard grading (i.e. when $A=\left[\begin{array}{lll}1 & \cdots & 1\end{array}\right]$ ), the module $M$ would be the homogeneous coordinate ring of the Veronese surface in $\mathbb{P}^{5}$. As a rational function, the Hilbert series of $M$ is

$$
\frac{F(\boldsymbol{t})}{\prod_{j}\left(1-\boldsymbol{t}^{\boldsymbol{a}_{j}}\right)}=\frac{\left(1-t_{1} t_{2}\right)\left(1-t_{1}^{2} t_{2}\right)\left(1-t_{1} t_{2}^{2}\right)\left(1+t_{1} t_{2}+t_{1}^{2} t_{2}+t_{1} t^{2}\right)}{\left(1-t_{1}^{2}\right)\left(1-t_{1} t_{2}\right)\left(1-t_{1}^{2} t_{2}\right)\left(1-t_{2}^{2}\right)\left(1-t_{1} t_{2}^{2}\right)\left(1-t_{1}^{2} t_{2}^{2}\right)} .
$$

Since $\ell:=\operatorname{codim}(M)=3>0$, it follows that $F(\mathbf{1})=0$.

In this context, an appropriate expansion of $F(\boldsymbol{t})$ comes from a choice of initial module. Each monomial initial module of $M$ is homogeneous with respect to the $\mathbb{Z}^{n}$-grading arising from the identity matrix $I_{n}$; see [MS, §2.2]. Since $M$ and any initial module have the same Hilbert series, the Taylor expansion for the numerator of the $\mathbb{Z}^{n}$-graded Hilbert series produces an expression for $F(\boldsymbol{t})$ as a polynomial in the variables $\left(1-\boldsymbol{t}^{\boldsymbol{a}_{1}}\right), \ldots,\left(1-\boldsymbol{t}^{\boldsymbol{a}_{n}}\right)$; see Exercise 8.15 in $\mathrm{MS}$. Moreover, the lowest terms in each such expression have degree $\ell$, are square-free, and have nonnegative coefficients; see Exercise 8.8 in [MS]. For instance, the monomial initial ideals $\left\langle x_{2}, x_{3}, x_{5}\right\rangle^{2}$ and $\left\langle x_{1}, x_{2}, x_{5}^{2}\right\rangle \cap\left\langle x_{2}^{2}, x_{5}, x_{6}\right\rangle$ of $I$ (chosen from among the 29 possibilities) yield the following expansions:

$$
\begin{aligned}
F(\boldsymbol{t})= & 4\left(1-\boldsymbol{t}^{\boldsymbol{a}_{2}}\right)\left(1-\boldsymbol{t}^{\boldsymbol{a}_{3}}\right)\left(1-\boldsymbol{t}^{\boldsymbol{a}_{5}}\right)-\left(1-\boldsymbol{t}^{\boldsymbol{a}_{2}}\right)^{2}\left(1-\boldsymbol{t}^{\boldsymbol{a}_{3}}\right)\left(1-\boldsymbol{t}^{\boldsymbol{a}_{5}}\right) \\
& -\left(1-\boldsymbol{t}^{\boldsymbol{a}_{2}}\right)\left(1-\boldsymbol{t}^{\boldsymbol{a}_{3}}\right)^{2}\left(1-\boldsymbol{t}^{\boldsymbol{a}_{5}}\right)-\left(1-\boldsymbol{t}^{\boldsymbol{a}_{2}}\right)\left(1-\boldsymbol{t}^{\boldsymbol{a}_{3}}\right)\left(1-\boldsymbol{t}^{\boldsymbol{a}_{5}}\right)^{2} \\
= & 2\left(1-\boldsymbol{t}^{\boldsymbol{a}_{1}}\right)\left(1-\boldsymbol{t}^{\boldsymbol{a}_{2}}\right)\left(1-\boldsymbol{t}^{\boldsymbol{a}_{5}}\right)+2\left(1-\boldsymbol{t}^{\boldsymbol{a}_{2}}\right)\left(1-\boldsymbol{t}^{\boldsymbol{a}_{5}}\right)\left(1-\boldsymbol{t}^{\boldsymbol{a}_{6}}\right) \\
& -\left(1-\boldsymbol{t}^{\boldsymbol{a}_{1}}\right)\left(1-\boldsymbol{t}^{\boldsymbol{a}_{2}}\right)\left(1-\boldsymbol{t}^{\boldsymbol{a}_{5}}\right)^{2}-\left(1-\boldsymbol{t}^{\boldsymbol{a}_{1}}\right)\left(1-\boldsymbol{t}^{\boldsymbol{a}_{2}}\right)\left(1-\boldsymbol{t}^{\boldsymbol{a}_{5}}\right)\left(1-\boldsymbol{t}^{\boldsymbol{a}_{6}}\right) \\
& -\left(1-\boldsymbol{t}^{\boldsymbol{a}_{2}}\right)^{2}\left(1-\boldsymbol{t}^{\boldsymbol{a}_{5}}\right)\left(1-\boldsymbol{t}^{\boldsymbol{a}_{6}}\right) .
\end{aligned}
$$


Observe that the coefficient of the term $\left(1-\boldsymbol{t}^{\boldsymbol{a}_{s_{1}}}\right)\left(1-\boldsymbol{t}^{\boldsymbol{a}_{s_{2}}}\right)\left(1-\boldsymbol{t}^{\boldsymbol{a}_{s_{3}}}\right)$ equals the multiplicity $\mu_{s}$ of the minimal prime $\left\langle x_{s_{1}}, x_{s_{2}}, x_{s_{3}}\right\rangle$ for the initial ideal; cf. Definition 8.43 in $\left[\mathrm{MS}\right.$. Since we have $\Phi_{r}\left[\left(1-\boldsymbol{t}^{\boldsymbol{a}_{i}}\right) F(\boldsymbol{t})\right]=\left(1-\boldsymbol{t}^{\boldsymbol{a}_{i}}\right) \Phi_{r}[F(\boldsymbol{t})]$, Theorem 1.1 gives

$$
\lim _{r \rightarrow \infty} \frac{\Phi_{r}[F(\boldsymbol{t})]}{r^{n-\ell-d}}=\frac{1}{(n-\ell-d) !} \sum_{\boldsymbol{s}}\left(\frac{\mu_{\boldsymbol{s}}}{m_{A_{\widehat{s}}-\ell}^{n-\ell-d}} K_{A_{\widehat{s}}}(\boldsymbol{t}) \prod_{1 \leqslant i \leqslant \ell}\left(1-\boldsymbol{t}^{\boldsymbol{a}_{s_{i}}}\right)\right),
$$

where $A_{\widehat{s}}$ is the $(d \times(n-\ell))$-submatrix of $A$ in which the columns indexed by $s$ are omitted and $m_{A_{\widehat{s}}}$ is the greatest common divisor of the maximal minors of $A_{\widehat{s}}$. In particular, we have

$$
\begin{aligned}
& \lim _{r \rightarrow \infty} \frac{\Phi_{r}[F(\boldsymbol{t})]}{r}=\frac{1}{2} t_{1} t_{2}\left(t_{1}+1\right)\left(t_{2}+1\right)\left(t_{1} t_{2}+1\right)\left(1-t_{1} t_{2}\right)\left(1-t_{1}^{2} t_{2}\right)\left(1-t_{1} t_{2}^{2}\right) \\
& =\frac{4}{4}\left(K_{\left[\begin{array}{lll}
2 & 0 & 2 \\
0 & 2 & 2
\end{array}\right]}(\boldsymbol{t})\right)\left(1-\boldsymbol{t}^{\boldsymbol{a}_{2}}\right)\left(1-\boldsymbol{t}^{\boldsymbol{a}_{3}}\right)\left(1-\boldsymbol{t}^{\boldsymbol{a}_{5}}\right) \\
& =\frac{2}{2}\left(K_{\left[\begin{array}{lll}
2 & 0 & 2 \\
1 & 2 & 2
\end{array}\right]}(\boldsymbol{t})\right)\left(1-\boldsymbol{t}^{\boldsymbol{a}_{1}}\right)\left(1-\boldsymbol{t}^{\boldsymbol{a}_{2}}\right)\left(1-\boldsymbol{t}^{\boldsymbol{a}_{5}}\right) \\
& +\frac{2}{2}\left(K_{\left[\begin{array}{lll}
2 & 2 & 0 \\
0 & 1 & 2
\end{array}\right]}^{(\boldsymbol{t})}\right)\left(1-\boldsymbol{t}^{\boldsymbol{a}_{2}}\right)\left(1-\boldsymbol{t}^{\boldsymbol{a}_{5}}\right)\left(1-\boldsymbol{t}^{\boldsymbol{a}_{6}}\right) .
\end{aligned}
$$

As the expansions for $F(\boldsymbol{t})$ vary, these equations lead to nontrivial relations among asymptotic $K$-polynomials associated to submatrices of $A$.

This approach also suggests a way to understand lower-order asymptotics. Specifically, if $\limsup _{r \rightarrow \infty} \frac{\Phi_{r}[F(\boldsymbol{t})]}{r^{n-\ell-d}}=\frac{G(\boldsymbol{t})}{(n-\ell-d) !}$, then $(n-\ell-d) ! \Phi_{r}[F(\boldsymbol{t})]-G(\boldsymbol{t})$ vanishes at $\boldsymbol{t}=\mathbf{1}$ and one should analyze $\limsup _{r \rightarrow \infty} \frac{(n-\ell-d) ! \Phi_{r}[F(\boldsymbol{t})]-G(\boldsymbol{t})}{r^{n-\ell-d-1}}$. Providing an algebraic or geometric interpretation for this lower-order asymptotics is an open problem.

Stochastic matrices. By scaling the matrix associated to the linear operator $\Phi_{r}$, we obtain a stochastic matrix. Specifically, the matrix $C(r):=r^{d-n}\left[C_{r}(\boldsymbol{u}, \boldsymbol{v})\right]$ where $\boldsymbol{u}$ and $\boldsymbol{v}$ range over $\operatorname{int}(Z) \cap \mathbb{Z}^{d}$ is a nonnegative square matrix each of whose columns sum to 1 . When $A=[1 \cdots 1]$, [Hol] (also see [DF1]) establishes that $C(r)$ has the following "amazing" properties:

- for all $r$, the stationary vector (i.e., the eigenvector with eigenvalue 1) corresponds to the coefficients of $K_{A}(\boldsymbol{t}) /(n-d)$ !;

- the matrix $C(r)$ has eigenvalues $r^{-i}$ for $0 \leqslant i<n-d$ with explicit eigenvectors independent of $r$;

- we have $C\left(r_{1}\right) C\left(r_{2}\right)=C\left(r_{1} r_{2}\right)$.

The next example illustrates how these properties extend to our more general setting.

Example 3.2. Let $A=\left[\begin{array}{ccccc}1 & 1 & 0 & 0 & -1 \\ 0 & 0 & 1 & 1 & 1\end{array}\right]$, so that

$$
Z=\operatorname{conv}\{(0,0),(2,0),(-1,1),(2,2),(-1,3),(1,3)\}
$$

and $K_{A}\left(t_{1}, t_{2}\right)=t_{1} t_{2}^{2}+2 t_{1} t_{2}+2 t_{2}^{2}+t_{2}$. Fixing $(1,2),(1,1),(2,0),(1,0)$ as the ordering for the interior lattice points yields the stochastic matrix

$$
C(r):=r^{-3}\left[\begin{array}{cccc}
\left(\begin{array}{c}
r+2 \\
3
\end{array}\right) & \left(\begin{array}{c}
r+1 \\
3
\end{array}\right) & \left(\begin{array}{c}
r+1 \\
3
\end{array}\right) & \left(\begin{array}{c}
r \\
3
\end{array}\right) \\
2\left(\begin{array}{c}
r+1 \\
3
\end{array}\right) & 2\left(\begin{array}{c}
r+1 \\
3
\end{array}\right)+\left(\begin{array}{c}
r+1 \\
2
\end{array}\right) & 2\left(\begin{array}{c}
r \\
3
\end{array}\right)+\left(\begin{array}{c}
r \\
2
\end{array}\right) & 2\left(\begin{array}{c}
r+1 \\
3
\end{array}\right) \\
2\left(\begin{array}{c}
r+1 \\
3
\end{array}\right) & 2\left(\begin{array}{c}
r \\
3
\end{array}\right)+\left(\begin{array}{c}
r \\
2
\end{array}\right) & 2\left(\begin{array}{c}
r+1 \\
3
\end{array}\right)+\left(\begin{array}{c}
r+1 \\
2
\end{array}\right) & 2\left(\begin{array}{c}
r+1 \\
3
\end{array}\right) \\
\left(\begin{array}{c}
r \\
3
\end{array}\right) & \left(\begin{array}{c}
r+1 \\
3
\end{array}\right) & \left(\begin{array}{c}
r+1 \\
3
\end{array}\right) & \left(\begin{array}{c}
r+2 \\
3
\end{array}\right)
\end{array}\right] .
$$


The eigenvalues of $C(r)$ are $1, r^{-1}, r^{-1}, r^{-2}$ and the corresponding eigenvectors are simply $\left[\begin{array}{llll}1 & 2 & 2 & 1\end{array}\right]^{\mathrm{t}},\left[\begin{array}{llll}1 & 0 & 0 & -1\end{array}\right]^{\mathrm{t}},\left[\begin{array}{llll}0 & 1 & -1 & 0\end{array}\right]^{\mathrm{t}},\left[\begin{array}{lllll}1 & -1 & -1 & 1\end{array}\right]^{\mathrm{t}}$.

In the standard graded case, $[\mathrm{Hol}]$ and [DF1] relate the matrix $C(r)$ to the process of "carries" when adding integers and to shuffling cards, respectively. Do our more general stochastic matrices also correspond to known Markov chains? Regardless, it would be interesting to bound the rates of convergence for the associated Markov chains and thereby extend the results in [DF2, §3].

\section{ACKNOWLEDGEMENTS}

The authors thank Matthias Beck, Daniel Erman, and Alan Stapledon for stimulating their interest in asymptotic Hilbert series and for providing feedback on a preliminary version of this document. The authors also thank Mike Roth for his valuable comments and insights. The computer software Macaulay2 [M2] was useful for generating examples. Both authors were partially supported by NSERC.

\section{REFERENCES}

[BS] Matthias Beck and Alan Stapledon, On the log-concavity of Hilbert series of Veronese subrings and Ehrhart series, Math. Z. 264 (2010), no. 1, 195-207. MR2564938 (2011a:05018)

[Bra] Petter Brändén, Polynomials with the half-plane property and matroid theory, Adv. Math. 216 (2007), no. 1, 302-320. MR2353258(2008h:05022)

[BHVW] Petter Brändén, James Haglund, Mirkó Visontai, and David G. Wagner, Proof of the monotone column permanent conjecture, Notions of positivity and the geometry of polynomials, Trends in Mathematics, Birkhäuser, Basel, 2011, pp. 63-78.

[Bre] Francesco Brenti, Log-concave and unimodal sequences in algebra, combinatorics, and geometry: an update, Jerusalem combinatorics '93, Contemp. Math., vol. 178, Amer. Math. Soc., Providence, RI, 1994, pp. 71-89. MR1310575 (95j:05026)

[BW] Francesco Brenti and Volkmar Welker, The Veronese construction for formal power series and graded algebras, Adv. in Appl. Math. 42 (2009), no. 4, 545-556. MR.2511015 (2010b:05190)

[BG] Winfried Bruns and Joseph Gubeladze, Polytopes, rings, and K-theory, Springer Monographs in Mathematics, Springer, Dordrecht, 2009. MR2508056 (2010d:19001)

[DF1] Persi Diaconis and Jason Fulman, Carries, shuffing, and an amazing matrix, Amer. Math. Monthly 116 (2009), no. 9, 788-803. MR2572087(2011d:60027)

[DF2] _ Carries, shuffing, and symmetric functions, Adv. in Appl. Math. 43 (2009), no. 2, 176-196. MR.2531920 (2010m:60028)

[EL] Lawrence Ein and Robert Lazarsfeld, Asymptotic syzygies of algebraic varieties, Invent. Math., published online 02 March 2012. DOI:10.1007/S00222-012-0384-5.

[Gar] Richard J. Gardner, The Brunn-Minkowski inequality, Bull. Amer. Math. Soc. (N.S.) 39 (2002), no. 3, 355-405. MR1898210 (2003f:26035)

[Hol] John M. Holte, Carries, combinatorics, and an amazing matrix, Amer. Math. Monthly 104 (1997), no. 2, 138-149. MR1437415 (98g:15034)

[M2] Daniel R. Grayson and Michael E. Stillman, Macaulay2, a software system for research in algebraic geometry, available at www.math.uiuc.edu/Macaulay2/.

[MS] Ezra Miller and Bernd Sturmfels, Combinatorial commutative algebra, Graduate Texts in Mathematics, vol. 227, Springer-Verlag, New York, 2005. MR2110098 (2006d:13001)

[Pos] Alexander Postnikov, Permutohedra, associahedra, and beyond, Int. Math. Res. Not. IMRN 6 (2009), 1026-1106. MR2487491 (2010g:05399)

[Sch] Alexander Schrijver, Theory of linear and integer programming, Wiley-Interscience Series in Discrete Mathematics, John Wiley \& Sons Ltd., Chichester, 1986. MR874114 (88m:90090)

[Sta1] Richard P. Stanley, Eulerian partitions of a unit cube, Higher combinatorics (Proc. NATO Advanced Study Inst., Berlin, 1976), Reidel, Dordrecht, 1977, p. 49. 
[Sta2] — Log-concave and unimodal sequences in algebra, combinatorics, and geometry, Graph theory and its applications: East and West (Jinan, 1986), Ann. New York Acad. Sci., vol. 576, New York Acad. Sci., New York, 1989, pp. 500-535. MR1110850 (92e:05124)

[Sta3] , Enumerative combinatorics. Vol. 1, Cambridge Studies in Advanced Mathematics, vol. 49, Cambridge University Press, Cambridge, 1997. MR.1442260 (98a:05001)

[Stu] Bernd Sturmfels, On vector partition functions, J. Combin. Theory Ser. A 72 (1995), no. 2, 302-309. MR 1357776 (97b:52014)

[Vil] Cédric Villani, Topics in optimal transportation, Graduate Studies in Mathematics, vol. 58, American Mathematical Society, Providence, RI, 2003. MR.1964483 (2004e:90003)

[Zie] Günter M. Ziegler, Lectures on polytopes, Graduate Texts in Mathematics, vol. 152, Springer-Verlag, New York, 1995. MR.1311028 (96a:52011)

35 Summerhill Road, Holland landing, Ontario, L9N 1C6, Canada

E-mail address: adam.r.mccabe@gmail.com

Department of Mathematics \& Statistics, Queen's University, Kingston, Ontario, K7L 3N6, CANADA

E-mail address: ggsmith@mast.queensu.ca 